\title{
The effectiveness of contrast-enhanced spectral mammography and magnetic resonance imaging in dense breasts
}

\author{
Wojciech Rudnicki ${ }^{1 A, B, C, D, E, F}$, Tomasz Piegza ${ }^{2 B}$, Natalia Rozum-Liszewska ${ }^{3 B}$, Mateusz Górski ${ }^{3 B}$, \\ Tadeusz J. Popiela ${ }^{4 E}$, Pawel Basta ${ }^{5 E}$, Sylwia Heinze ${ }^{2 C}$, Elzbieta Luczynska ${ }^{6 E, F}$ \\ 'Electroradiology Department, Faculty of Health Sciences, Jagiellonian University Collegium Medicum, Krakow, Poland \\ ${ }^{2}$ Maria Sklodowska-Curie National Research Institute of Oncology in Cracow, Poland \\ ${ }^{3}$ University Hospital in Krakow, Krakow, Poland \\ ${ }^{4}$ Chair of Radiology, Jagiellonian University Medical College, Krakow, Poland \\ ${ }^{5}$ Jagiellonian University Medical College, Krakow, Poland \\ ${ }^{6}$ Institute of Medical Sciences, Medical College of Rzeszow University, Rzeszow, Poland
}

\section{Abstract}

Purpose: Breast cancer is the most common cause of death from neoplastic disease in women. Among all breast anatomy types, glandular type is the most problematic concerning evaluation. While digital mammography still remains the basic diagnostic tool, one must be aware of its limitations in dense breasts. Although magnetic resonance imaging (MRI) has greatly improved sensitivity, its specificity is low. Moreover, there are contraindications for MRI for some patients, so a substitute has been searched for. This study was performed to check if contrast-enhanced spectral mammography (CESM) can be a viable option for patients with dense breasts.

Material and methods: The study involved 121 patients with abnormalities detected on base-line diagnostic imaging (ultrasound or mammography). The patients had subsequent examinations, both CESM and MRI performed within a maximum 2-month time interval. The sensitivity and specificity of both methods in the whole group as well as in specific breast structure types were measured and compared.

Results: Contrast enhancement was visible in all 121 cases on MRI, while on CESM lack of enhancement was noted in 13 cases. All of those 13 lesions turned out to be benign. There were 40 (33\%) benign and 81 (69\%) malignant tumours. The analysed group included 53 (44\%) glandular type breast patients, 39 (32\%) mixed type, and 29 (23\%) fatty type. Although MRI proved to be slightly more effective in dense breasts, both methods showed similar results in the whole study group.

Conclusion: CESM can be used with confidence in patients with glandular breast type when MRI is not available or there are reported contraindications to MRI.

Key words: cancer, breast, diagnostic imaging, MRI, CESM.

\section{Introduction}

Breast cancer is the most commonly diagnosed cancer in women [1]. Mammography remains the standard method in breast cancer diagnostics. Despite the development of different diagnostic methods, only mammographic sen- sitivity and specificity in clinically asymptomatic cancer detection have been confirmed [2]. Ultrasound is still considered to be a complementary examination to mammography. Sensitivity and specificity of mammography for breast cancer detection are defined [3-6]. However, sensitivity is particularly low in the case of glandular

Correspondence address:

Wojciech K. Rudnicki, Department of Electroradiology, Jagiellonian University Medical College, Krakow, Poland, e-mail: wrudnicki@cmuj.pl

Authors' contribution:

A Study design · B Data collection · C Statistical analysis · D Data interpretation · E Manuscript preparation · F Literature search · G Funds collection 
dense breast tissue; it can be as low as $30-65 \%$ [4; 7] for 2 main reasons: tissue overlapping and the fact that X-ray absorption of glandular tissue is similar to that of a cancer, making it indistinguishable. Thus, new diagnostic methods enabling detection of greater numbers of suspicious breast lesions have been developed. Among them, the 2 latest techniques are based on mammography: contrastenhanced spectral mammography (CESM) and digital breast tomosynthesis (DBT). Magnetic resonance imaging (MRI) is an entirely independent new method [8].

The role of MRI in breast cancer diagnostics has been confirmed. Based on the literature data it is known that while the sensitivity of MRI is high, its specificity remains low $[9,10]$. Another limitation of this method is the time taken to perform the examination: 25-30 min, whereas CESM lasts only about $10 \mathrm{~min}$. What is more, CESM examination produces 4 images that are easy to interpret. Conversely, the number of sequences acquired on MRI is large, which considerably prolongs the interpretation time. According to the literature data, CESM and MRI are comparable in terms of diagnostic efficiency $[9 ; 11]$. However, there are no sufficient data pertaining to CESM and MRI comparison in patients with glandular breast types. Additionally, one of the studies showed that high-risk patients would prefer CESM instead of MRI as a screening examination if their sensitivity was similar [12]. In order to increase the number of patients who can be provided with the newest and most accurate diagnostic imaging service, and to ensure maximum comfort to a patient during the diagnostic period, it is necessary to understand which imaging methods are substitutable. This study was performed to clarify whether CESM can be used as a viable option for patients with dense breasts.

\section{Material and methods}

This retrospective study included a group of 121 patients with abnormalities found on basic examinations: ultra- sound (US) or/and digital mammography (DM). Subsequently, CESM or MRI examinations were performed. Two imaging methods were used when one of them did not give sufficient clinical information and the clinical risk of cancer was high. All of the patients had to undergo both CESM and MRI followed by histological examination of the lesions to be included in the study group.

Figure 1 shows the enhancement of the lesions on CESM and MRI. It is worth noticing that the lesion was not visible on images without contrast administration.

CESM was performed on a GE Senographe Essential system with a standard protocol used in the facility $2 \mathrm{~min}$ after administration of Iopromide $(1.5 \mathrm{ml} / \mathrm{kg}$ of body weight) the first acquisition of mammography image was performed. The examination sequence started with a breast without any lesions found in previous examinations All projections were acquired within 7 minutes of contrast administration [13]. The examination was followed by image annotation by a radiologist with at least 5 years of experience in CESM studies. Lesion enhancement, if visible, was described qualitatively as weak, medium, or strong. The margin and pattern of enhancement were also evaluated. The margin was described as firm or blurred, while the pattern as homogenous or heterogeneous. Breast type was defined on low energy images as one of the following: fatty, mixed, or glandular (Figure 2).

MRI examination was performed on a $1.5 \mathrm{~T}$ Siemens Avanto system. Patients were scheduled for breast MRI during the second week of their menstrual cycle (5-12 day) to achieve the best sensitivity. Acquired sequences included T1, T2, and T2 with Fat Saturation, diffusion-weighted images (DWI), and dynamic series after contrast medium administration (gadobutrol in a dose of $0.1 \mathrm{ml} / \mathrm{kg}$ of body weight). The next step included image annotation by a radiologist with at least 5 years of experience in breast MRI. If any enhancing lesions were visible, a kinetic curve was analysed and assessed as persistent, plateau, or washout.
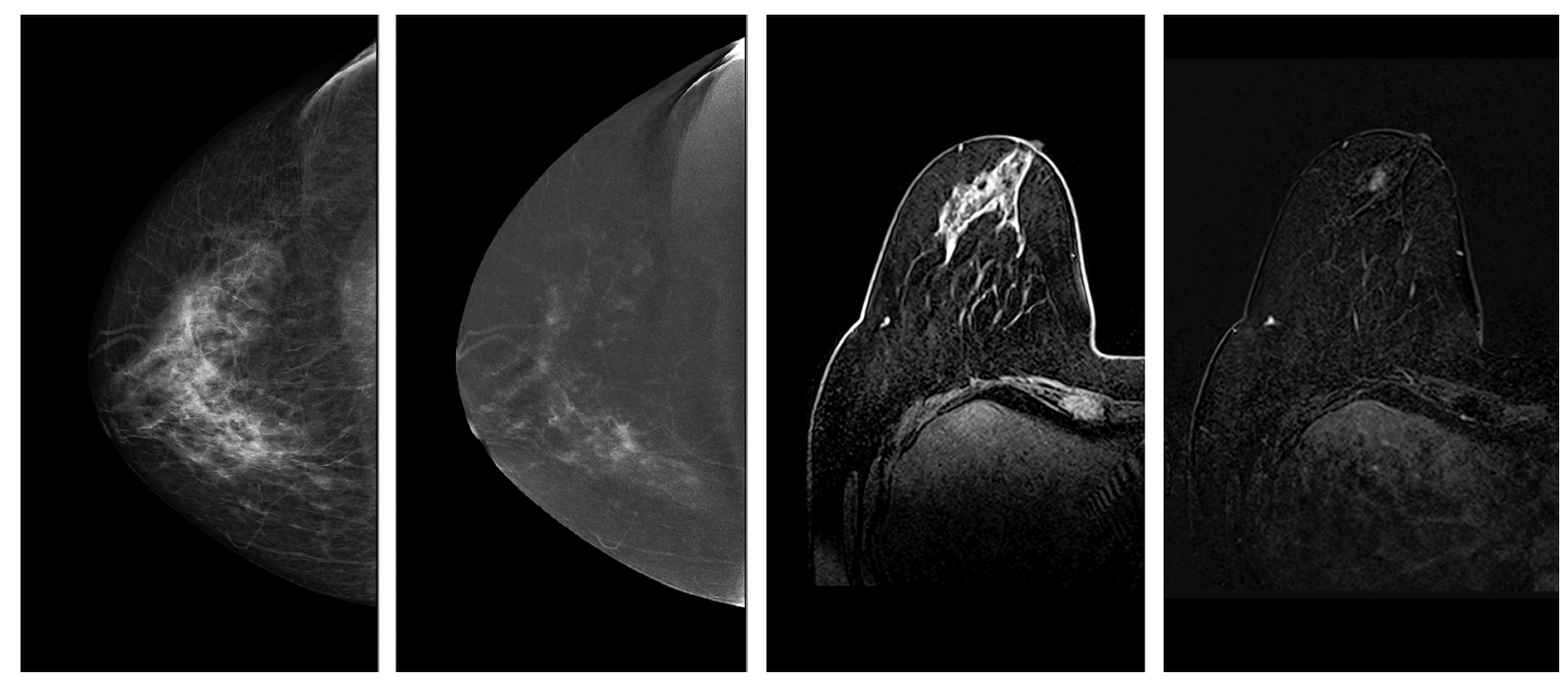

Figure 1. From the left: low-energy (contrast-enhanced spectral mammography - CESM), subtraction images (CESM), T1Fat Sat C+ image (magnetic resonance imaging $-\mathrm{MRI}$ ) and subtraction T1 Fat Sat $\mathrm{C}+$ of the right breast of a patient with suspicious lesion 

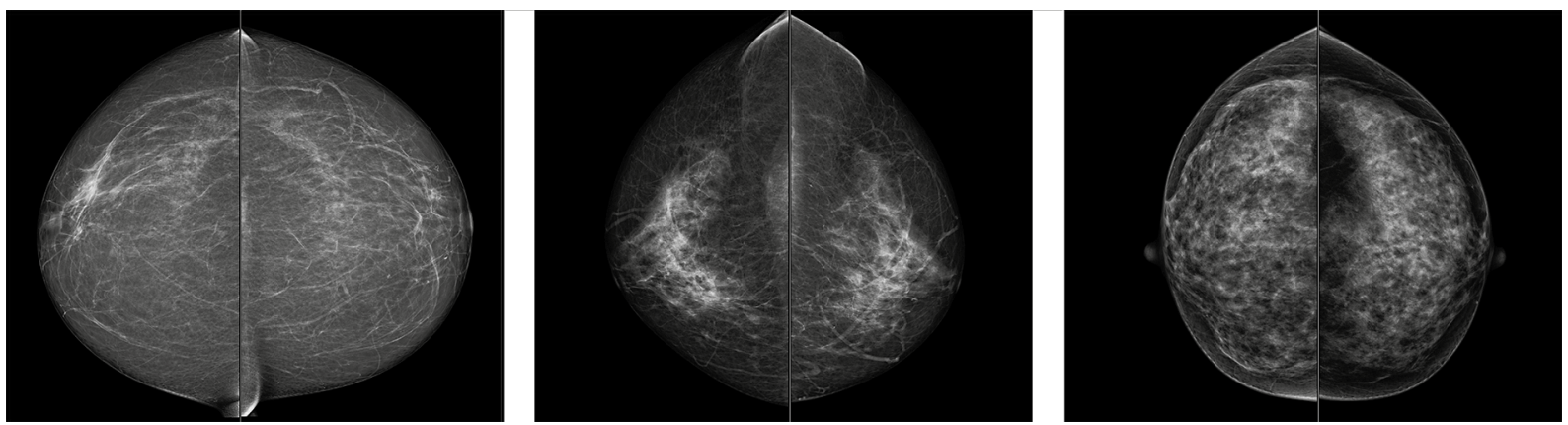

Figure 2. Breast type on contrast enhanced spectral mammography seen on low-energy images. Cranio-caudal projections of both breasts. From the left side: fatty, mixed, and glandular type
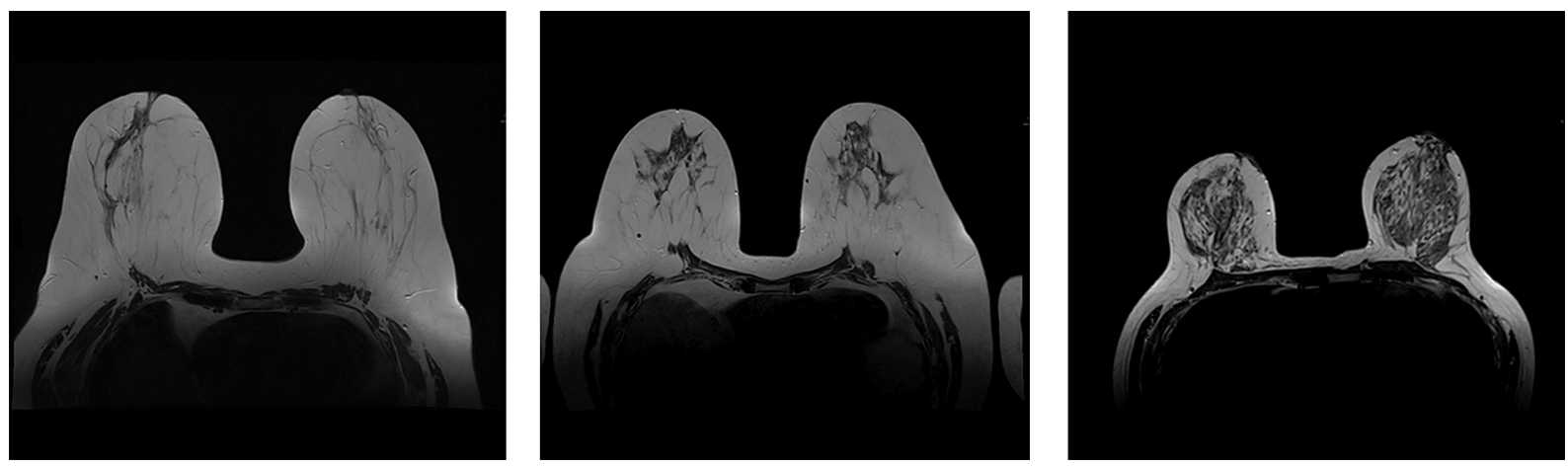

Figure 3. Breast type on magnetic resonance imaging T2-weighted images. From the left side: fatty, mixed, and glandular type. Images of the same patients as in Figure 2

In some cases, the curve was described as not specified/ characteristic if it did not match the criteria mentioned before. The breast type was defined on T2WI as one of the following: fatty, mixed, or glandular (Figure 3).

Histopathological examination of all lesions was performed. The material was obtained by either core-needle biopsy (CNB), vacuum-assisted breast biopsy (VABB) guided by US, DM, or MRI, or lumpectomy. Pathologists performing the examination had over 10 years of experience in breast cancer cases.

Statistical analysis was performed using Statistica software. Test characteristics including sensitivity, specificity, positive predictive value, and negative predictive value for both CESM and MRI examinations were evaluated to compare the effectiveness of both methods in detecting breast lesions; ROC analysis was performed to check if both CESM and MRI can be used to distinguish benign and malignant lesions.

This study was performed in compliance with the Declaration of Helsinki, and it received the approval of the Ethical Committee at the Regional Medical Chamber (acceptance No. OIL/KBL/17/2018).

\section{Results}

Histopathological examination of all lesions found on all diagnostic examinations revealed following results: $81(69 \%)$ cancerous lesions in the whole group, including $10(8 \%)$ non-infiltrating cancers, and 71 (59\% of the whole) infiltrating cancers. The remaining 40 (33\%) lesions were benign. In 13 patients no enhancement was visible, except parenchymal BPE on CESM. Those lesions were classified as BI-RADS 1 and none of them was cancerous on histopathology. On MRI the enhancement was visible in the suspicious lesion area in all cases, and 9 of them were described as benign (BI-RADS 2). On histopathology the lesions were benign.

The breast anatomy type of the patients with the above diagnosed lesions was predominantly glandular - 53 (44\%), mixed - 39 (32\%), and the rarest - fatty - 29 (23\%).

Contrast enhancement characteristics were analysed both for CESM and MRI for the whole group of patients and separately for the group of patients with glandular breast type. Features such as margin and pattern were statistically insignificant and were not taken into consideration.

Enhancement intensity on CESM and kinetic curve type on MRI appeared to be important elements indicating a relationship between CESM and MRI.

\section{Enhancement intensity on contrast-enhanced spectral mammography}

Among the lesions analysed on CESM strong enhancement was seen the most frequently - in 46 (43\%) cases, followed by medium - $34(31 \%)$ cases, and weak enhancement - in $28(26 \%)$ cases.

The division of cases depended on enhancement intensity on CESM $(p=0.012)$. Strong enhancement was applied 


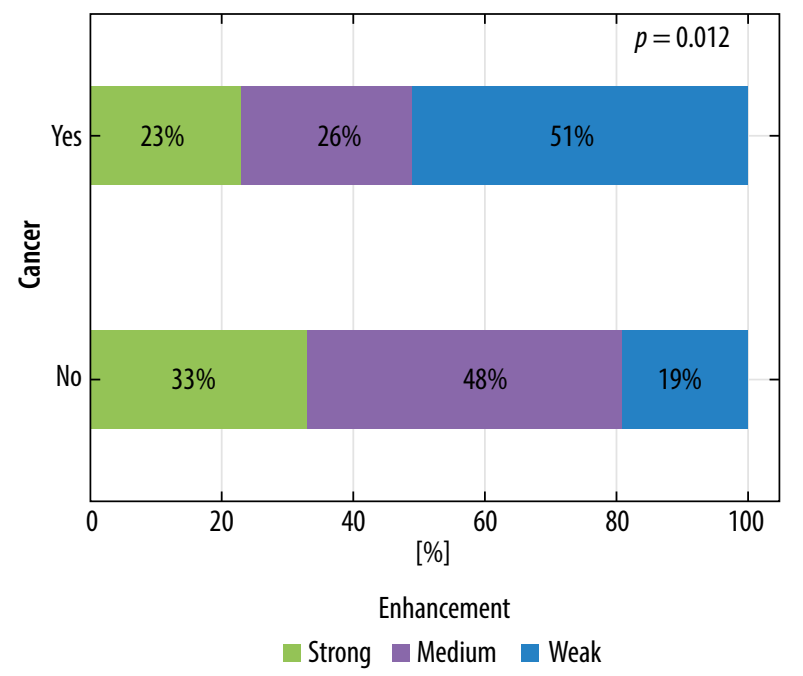

Figure 4. Distribution of enhancement intensity on contrast-enhanced spectral mammography according to lesion type

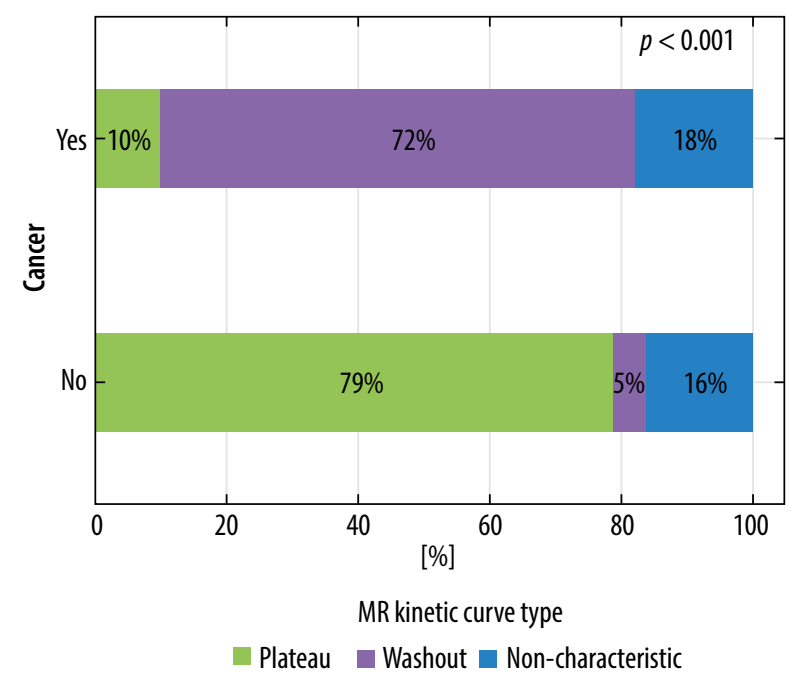

Figure 6. Distribution of enhancement curve on magnetic resonance imaging according to lesion type

more often in case of cancerous lesions than among noncancerous lesions (51\% and 19\%, respectively). The results are depicted in Figure 4.

If the lesions were diagnosed in glandular breast type strong enhancement on CESM was applied in $22(44 \%)$ cases, medium in 15 (30\%) cases, and weak in 13 (26\%) cases. The lesion type depended on enhancement intensity visible on CESM $(p=0.006)$. The results are depicted in Figure 5.

\section{Kinetic curve type on magnetic resonance imaging}

Among the described lesions the main enhancement curve type on MRI was washout - 60 (50\%) cases, followed by non-characteristic curve in $32 \%$ (38 cases), and plateau in $18 \%$ ( 21 cases). Persistently enhancing (progressive) curve was seen in 2 cases, 1 of them in glandular breast type. Curve type on MRI is strongly correlated with

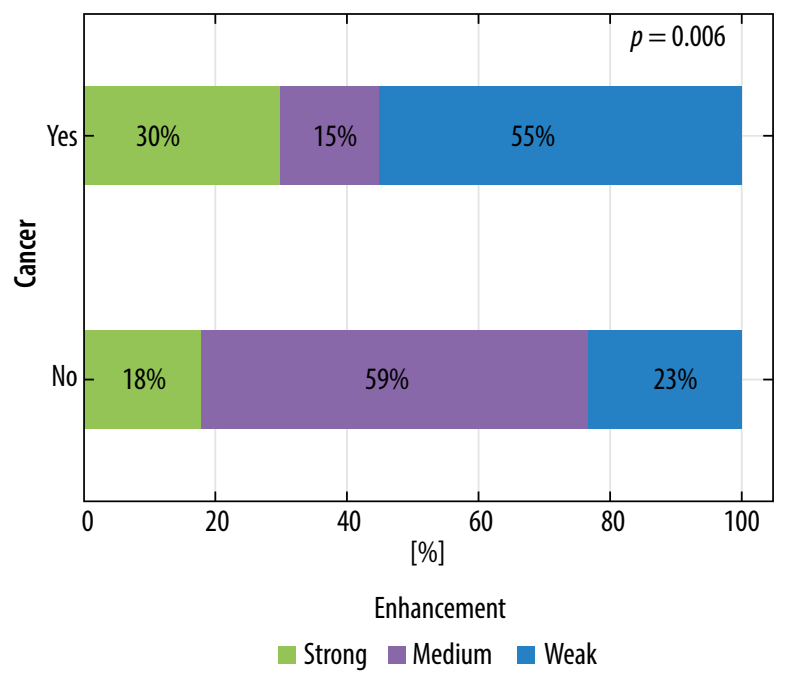

Figure 5. Distribution of enhancement intensity on contrast-enhanced spectral mammography according to lesion type in dense breast

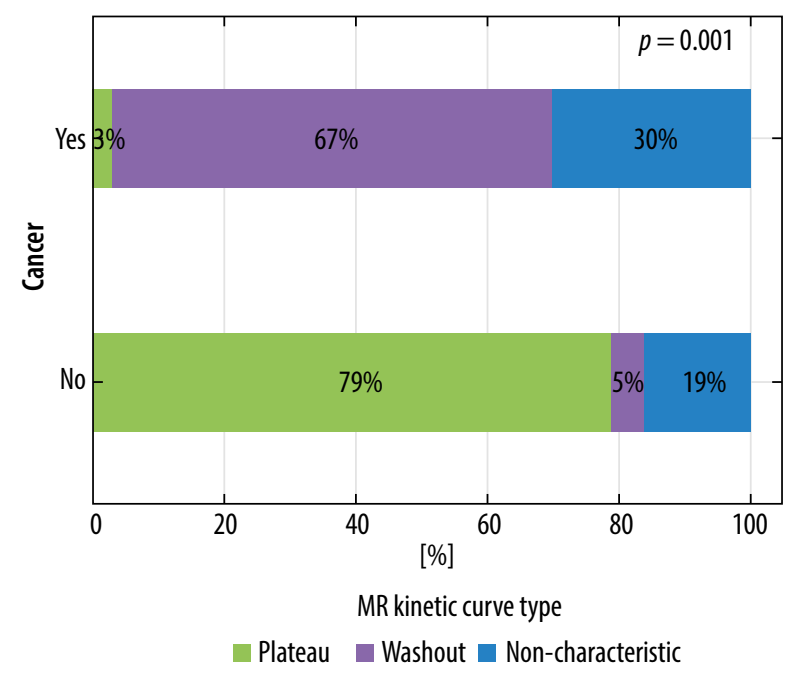

Figure 7. Distribution of enhancement curve on magnetic resonance imaging according to lesion type in dense breast

lesion type $(p<0.001)$. Washout type curve is the most common for cancerous lesions, and non-characteristic type for non-cancerous lesions. The correlations are depicted in Figure 6. The results were the same for the lesions diagnosed in dense breasts (Figure 7).

\section{Sensitivity and specificity}

Obtained data enabled the assessment of sensitivity, specificity, and accuracy of both methods. Their PP and NP values were also defined. The results are presented in Table 1.

Similar analysis was performed considering only dense breasts. The results are presented in Table 2 .

ROC analysis confirmed that both methods can be applied to differentiate between benign and malignant lesions (AUC > 0.5, $p<0.05$ ). Comparing AUC results for all breast anatomy types it is not possible to indicate the 
Table 1. Diagnostic test results for contrast-enhanced spectral mammography and magnetic resonance imaging

\begin{tabular}{|l|c|c|c|c|c|}
\hline & Sensitivity & Accuracy & Specificity & PPV & NPV \\
\hline CESM & $100 \%$ & $77 \%$ & $33 \%$ & $75 \%$ & $100 \%$ \\
\hline MRI & $100 \%$ & $74 \%$ & $23 \%$ & $72 \%$ & $100 \%$ \\
\hline
\end{tabular}

Table 2. Diagnostic test results for contrast-enhanced spectral mammography and magnetic resonance imaging in dense breasts

\begin{tabular}{|l|c|c|c|c|c|} 
& Sensitivity & Accuracy & Specificity & PPV & NPV \\
\hline CESM & $100 \%$ & $68 \%$ & $15 \%$ & $66 \%$ & $100 \%$ \\
\hline MRI & $100 \%$ & $72 \%$ & $25 \%$ & $69 \%$ & $100 \%$ \\
\hline
\end{tabular}

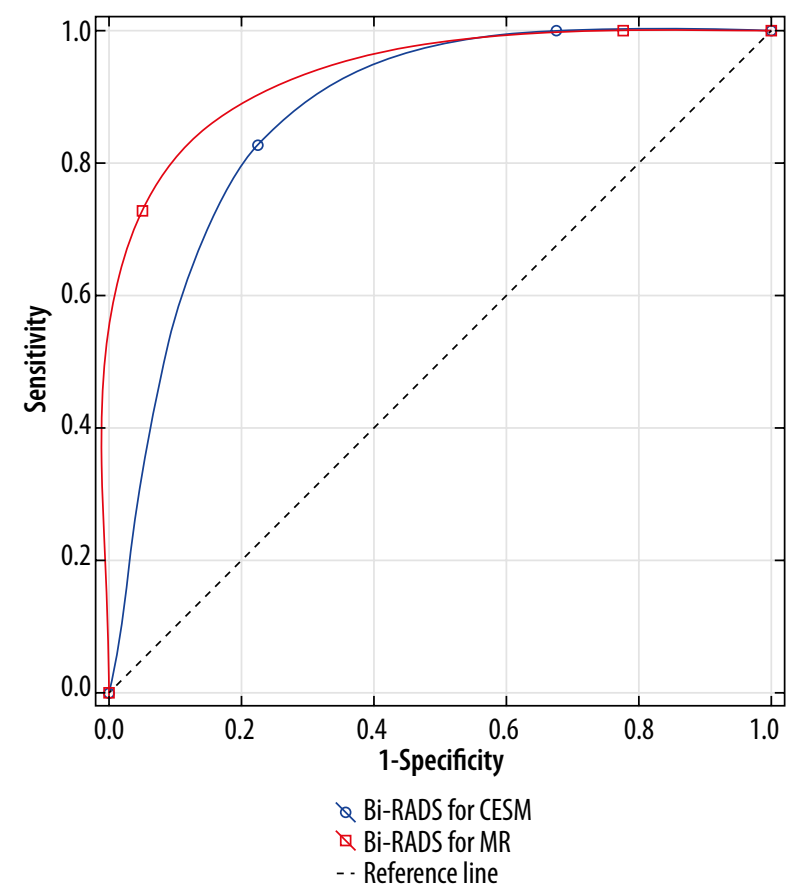

Figure 8. Receiver operating characteristic curves for contrast-enhanced spectral mammography and magnetic resonance imaging

more effective method, $p=0.32$. The results are illustrated in Figure 8.

For lesions diagnosed in dense breasts differentiation between benign and malignant lesions was also possible in both methods (AUC $>0.5$ and $p<0.05$ ). Comparison of the results revealed that MRI was more effective in malignant lesion detection $(p=0.005)$. The results are illustrated in Figure 9.

\section{Discussion}

According to the literature data, sensitivity and specificity of spectral mammography are comparable to those of MRI $[11,14]$. Some authors state that the sensitivity of MRI is slightly better than the sensitivity of CESM [15], whereas others state the opposite [16]. In our study the enhancement intensity on CESM was compared with enhancement intensity on MRI (curves evaluation) as well as the sensitivity and specificity of both methods in dense

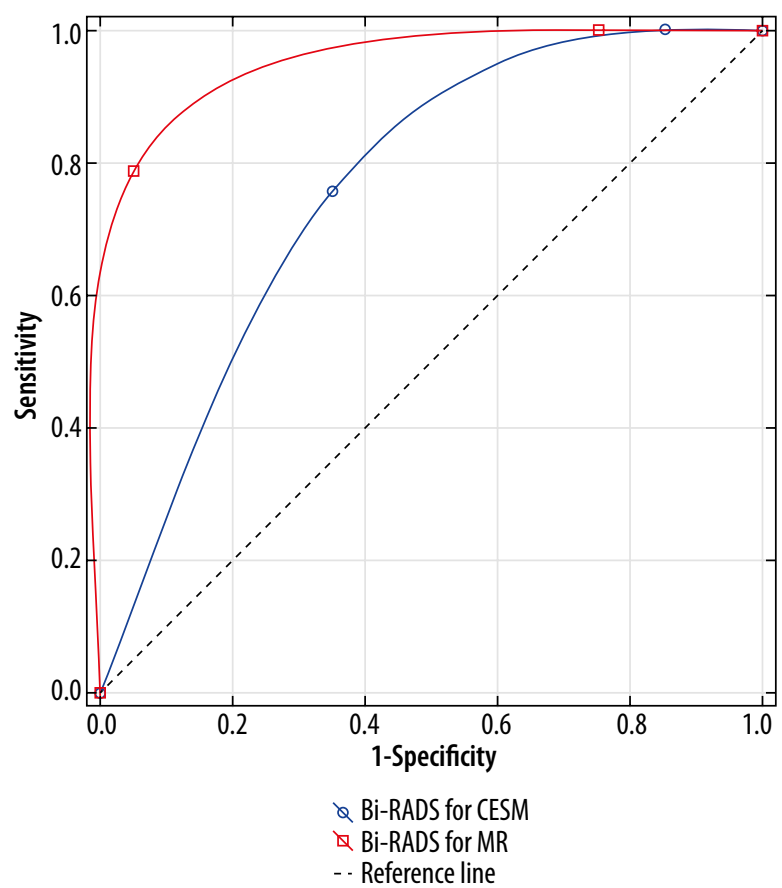

Figure 9. Receiver operating characteristic curves for contrast-enhanced spectral mammography and magnetic resonance imaging in dense breasts

breasts. Material analysis demonstrated both CESM and MRI sensitivity in dense breasts to be $100 \%$. Until now, only the general sensitivity and specificity of both methods have been studied and presented in the literature, but their efficiency in dense breast remained undetermined.

Our study showed that CESM is a great diagnostic tool not only for patients with fatty type breast but also for those with glandular type. Therefore, MRI and CESM can be substitutes for each other in cases where contraindications appear for one of the modalities. The morphology of the lesions found on CESM in dense breasts is the same as of those in fatty type breasts, thus no special training is required for reading radiologists. This is a great added value because most of the lastest mammography machine models are capable of acquiring CESM images after a software upgrade, which is much cheaper and less space consuming than MRI scanner installation. It also provides the opportunity to diagnose more patients for smaller Breast Care Centres in their own facilities. 
However, there are some limitations of the technique and of the study itself. CESM is related with exposition to ionic radiation, which constitutes a contraindication for patients with BRCA mutations as well as pregnant women. Iodine-based contrast medium causes more allergic reactions than gadolinium-based agents. In both CESM and MRI the kidney function needs to be monitored in patients with kidney diseases. CESM can be performed despite cardiac stimulators and any other metallic implants, unlike in MRI. The examination time of CESM is shorter and it does not require the patients to maintain a prone position for a long time, which can be problematic for those with spine diseases. There is also no gantry, which can cause claustrophobic symptoms and is a limitation for obese patients or patients with large breasts.

As for the study limitations, the studied group was small; this results from the fact that undergoing both CESM and MRI can extend the diagnostic part of the treatment process for patients. Moreover, because there are no guidelines for CESM pertaining to the adequate time of menstrual cycle, the patients were not distinctively scheduled. The literature data shows that this issue can be neglected in the overall exam review $[9 ; 17]$.

\section{Conclusions}

CESM can be used with confidence instead of MRI for cancer detection in patients with dense breast. It provides the same sensitivity as MRI, but with negligibly lower specificity than MRI. More studies are needed to confirm the obtained results.

\section{Acknowledgements}

Authors express their gratitude for PhD Dorota Olszewska, who assisted in our statistical calculations.

\section{Conflict of interest}

The authors report no conflict of interest.

\section{References}

1. Ganz PA, Goodwin PJ. Breast cancer survivorship: where are we today? Adv Exp Med Biol 2015; 862: 1-8.

2. Drukteinis JS, Mooney BP, Flowers CI, Gatenby RA. Beyond mammography: new frontiers in breast cancer screening. Am J Med 2013; 126: 472-479.

3. Zeeshan M, Salam B, Khalid QSB, Alam S, Sayani R. Diagnostic accuracy of digital mammography in the detection of breast cancer. Cureus 2018; 10: e2448.

4. Bell RJ. Mammographic density and breast cancer screening. Climacteric 2020; 23: 460-465.

5. Hollingsworth AB. Redefining the sensitivity of screening mammography: a review. Am J Surg 2019; 218: 411-418.

6. Shen Y, Zelen M. Screening sensitivity and sojourn time from breast cancer early detection clinical trials: mammograms and physical examinations. J Clin Oncol 2001; 19: 3490-3499.

7. Weigel S, Heindel W, Heidrich J, Hense HW, Heidinger O. Digital mammography screening: sensitivity of the programme dependent on breast density. Eur Radiol 2017; 27: 2744-2751.

8. Bidlek M, Kovács E, Fehér K, Gõdény M. New trends and novel possibilities in the diagnostic imaging of breast cancer. Magy Onkol 2015; 59: 44-55 [Article in Hungary].

9. Fallenberg EM, Schmitzberger FF, Amer H, et al. Contrast-enhanced spectral mammography vs. mammography and MRI - clinical performance in a multi-reader evaluation. Eur Radiol 2017; 27: 2752 2764.

10. Roganovic D, Djilas D, Vujnovic S, Pavic D, Stojanov D. Breast MRI, digital mammography and breast tomosynthesis: comparison of three methods for early detection of breast cancer. Bosn J Basic Med Sci 2015; 15: 64-68.
11. Li L, Roth R, Germaine P, et al. Contrast-enhanced spectral mammography (CESM) versus breast magnetic resonance imaging (MRI): a retrospective comparison in 66 breast lesions. Diagn Interv Imaging 2017; 98: 113-123.

12. Phillips J, Miller MM, Mehta TS, et al. Contrast-enhanced spectral mammography (CESM) versus MRI in the high-risk screening setting: patient preferences and attitudes. Clin Imaging 2017; 42: 193197.

13. Puong S, Bouchevreau X, Patoureaux F, Iordache R, Muller S. Dualenergy contrast enhanced digital mammography using a new approach for breast tissue cancelling. Proc. SPIE 6510, Medical Imaging 2007: Physics of Medical Imaging, 65102H (21 March 2007); https:// doi.org/10.1117/12.710133

14. Xiang W, Rao H, Zhou L. A meta-analysis of contrast-enhanced spectral mammography versus MRI in the diagnosis of breast cancer. Thorac Cancer 2020; 11: 1423-1432.

15. Lee-Felker SA, Tekchandani L, Thomas M, et al. Newly diagnosed breast cancer: comparison of contrast-enhanced spectral mammography and breast MR imaging in the evaluation of extent of disease. Radiology 2017; 285: 389-400.

16. Wang Q, Li K, Wang L, Zhang J, Zhou Z, Feng Y. Preclinical study of diagnostic performances of contrast-enhanced spectral mammography versus MRI for breast diseases in China. Springerplus 2016; 5: 763 .

17. Zhao S, Zhang X, Zhong H, et al. Background parenchymal enhancement on contrast-enhanced spectral mammography: influence of age, breast density, menstruation status, and menstrual cycle timing. Sci Rep 2020; 10: 8608. 